\title{
Measures to Assure that Ebola Guidelines Are Correctly Applied
}

\author{
José Joaquín Mira ${ }^{a}$ b Alberto Asencio-Aznar ${ }^{c}$ Teresa Gea $^{d}$ Susana Lorenzo ${ }^{\mathrm{e}}$ \\ Pedro Antón ${ }^{f}$ \\ a Universidad Miguel Hernández and b Alicante-Sant Joan Health District, Alicante, ${ }^{c}$ Alicante-Sant Joan Health \\ District, Mutxamel, ${ }^{d}$ Preventive Medicine Unit, Hospital Sant Joan D'Alacant, Alicante, ${ }^{e}$ Hospital Universitario \\ Fundación Alcorcón, Madrid, and ${ }^{\mathrm{f}}$ Hospital Sant Joan D'Alacant, Alicante, Spain
}

\section{Key Words}

Ebola virus · Protocol · Occupational health · Primary care

\begin{abstract}
Objective: The purpose of this study was to assure the application, in our primary care health district, of the World Health Organisation and Spanish Ebola virus disease (EVD) guidelines, and to identify and resolve gaps, in order to protect professionals from the risks associated with attending to patients with Ebola. Materials and Methods: A checklist was drawn up and an audit procedure of the structural, functional and operational conditions was designed for managing a self-presenting patient suspected with EVD in general practice. This audit was applied twice (before and after assessment) in 9 health centres of a health district in Spain. The checklist was structured according to 9 categories (51 elements in total). This tool was designed to avoid errors in the management of EVD patients that are considered very serious in view of their potential consequences. Results: Of the 51 elements on the checklist, 7-26 (14-51\%) required direct intervention to rectify deficits in the first audit. The following elements requiring improvement were identified: the incorporation of memory aids to ensure the correct application of the EVD protocol, defining what information should be provided to the patient and (where applicable) anyone accom-
\end{abstract}

\begin{tabular}{ll}
\hline KARGER & $\begin{array}{l}\text { ( } 2015 \text { S. Karger AG, Basel } \\
1011-7571 / 15 / 0252-0191 \$ 39.50 / 0\end{array}$ \\
E-Mail karger@karger.com & $\begin{array}{l}\text { This is an Open Access article licensed under the terms of the } \\
\text { www.karger.com/mpp }\end{array}$ \\
$\begin{array}{l}\text { Creative Commons Attribution-NonCommercial 3.0 Un- } \\
\text { ported license (CC BY-NC) (www.karger.com/OA-license), } \\
\text { applicable to the online version of the article only. Distribu- } \\
\text { tion permitted for non-commercial purposes only. }\end{array}$
\end{tabular}

panying them as well as incorporating advice on how to deal with any individuals with whom they might have come into contact. A second audit assured the adequacy of the proposed solutions. Conclusion: In this study, the tool achieved the successful application of EVD protocol, assuring that primary care centres were able to handle an Ebola suspect patient safely.

(c) 2015 S. Karger AG, Basel

\section{Introduction}

The first reports of Ebola virus disease (EVD or simply 'Ebola') were in 1976 [1, 2]. The virulence of the most recent outbreak in 2014 (with more deaths than on previous occasions) and the spread of Ebola to Europe and the USA were the major differences between the 2014 and 1976 outbreaks.

The first case of EVD diagnosed outside Africa was on the 6th October 2014 [3-5]. After that, cases of secondary infection were also reported in the USA, and several people working in the care of EVD patients were treated in European and US hospitals [6, 7].

The modes of transmission of Ebola and the precautions that professionals should take have already been reported [8]. On the 8th August 2014, the World Health

Prof. José Joaquín Mira

Universidad Miguel Hernández, Altamira Building

Avd Universidad s/n, Elche

ES-03202 Alicante (Spain)

E-Mail jose.mira@umh.es 
Organisation (WHO) declared the EVD outbreak a Public Health Emergency of International Concern. In Spain, like in other countries, this alert resulted in providing education and training about EVD to all health-care professionals. However, a case of secondary infection of a Spanish health-care worker who had treated an Ebola patient caused alarm among Spanish health-care professionals $[9,10]$. The staff felt personally threatened and healthcare institutions intensified their training and reviewing of procedures, so as to be sure they were able to cope with this challenge. A review of safe systems of working to protect staff was required to recover confidence in the EVD procedure $[7,11]$.

Although the majority of approaches and procedures were developed for inpatients, anybody that had been in contact with individuals with Ebola or in risk zones that presented clinical symptoms of EVD (fever, vomiting, bleeding or diarrhoea) could receive medical attention in settings other than designated Ebola care facilities [12]. The objective of this study was to ensure the applicability of the WHO and Spanish EVD guidelines in our primary care health district, and to identify and resolve gaps, so as to protect health-care professionals from the risks associated with attending to EVD patients.

\section{Materials and Method}

The most recent recommendations from the WHO [13] and Spanish Ministry of Health [14] in relation to Ebola constituted the base for the EVD protocols applied in Spain. When the Public Health Emergency was declared by the WHO, Spanish hospitals and primary care centres reinforced the training of health-care professionals and provided resources to cope with the Ebola crisis according to the recommendations of Public Health authorities. Hospitals and primary care centres developed procedures to apply the Spanish Ebola guidelines which included the WHO recommendations. They provided resources and training to cope with EVD, considering the occupational health of health-care professionals a priority. Hospitals were the focus of measures for a safer context, as in primary care there were expected gaps in the resources and information received. With a view to ensuring the appropriate implementation of the WHO and Spanish Ministry of Health recommendations, a checklist and audit procedure were designed with regard to the structural, functional and operational procedures for managing a self-presenting patient suspected to have Ebola in the primary health-care context. The checklist was designed for the purpose of the audit but also as a tool for the staff members of primary care centres to use in order to increase confidence and safety.

For drawing up the checklist, a working group was set up in the Alicante-San Joan Health District comprising professionals working in hospitals and primary care centres. This team included managers, public health officials, general practitioners and quality staff. Our health district attends to a total of 219,871 inhabitants at 9

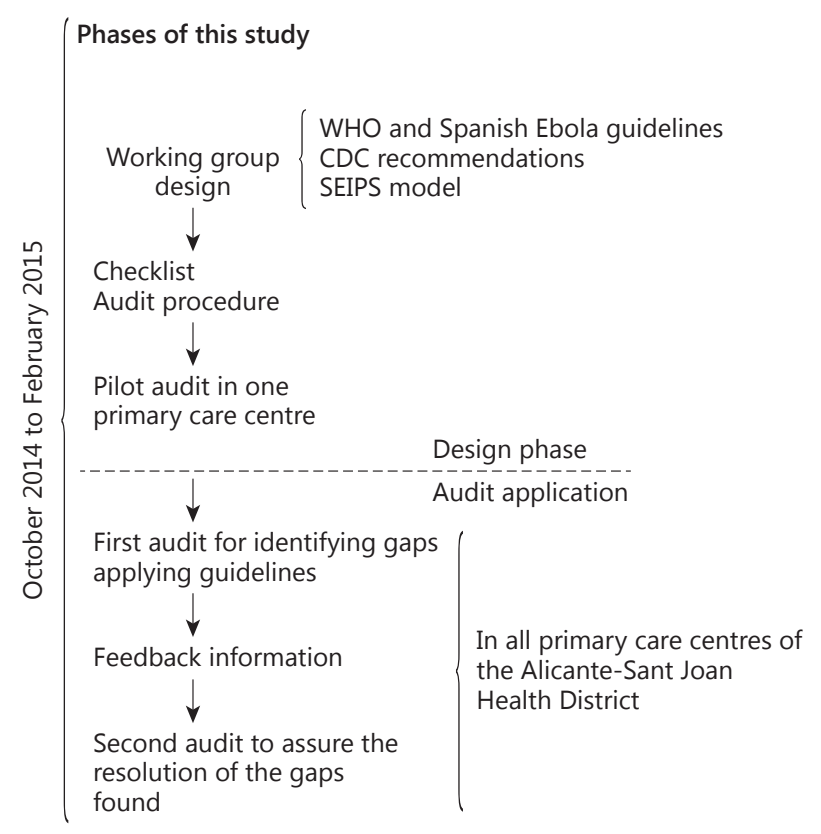

Fig. 1. Diagram of the procedure followed in this study.

health centres with the following ratios: 0.63 physicians (including paediatricians) to 1,000 inhabitants and 0.52 nurses to 1,000 inhabitants. Audits were carried out in all the health centres of this health district.

In the design phase of the checklist, the working group first reviewed the material from the Centers for Disease Control (CDC) and Prevention in the USA [15] (i.e. the Detailed Hospital Checklist for Ebola Preparedness) and the SEIPS model [16] (Systems Engineering Initiative for Patient Safety), devised for the redesigning of systems and taking into account the human factor.

The checklist was designed to reduce the incidence of the most serious errors (fig. 1). The following categories were considered: accessibility to information and the Spanish EVD protocol (including the WHO recommendations), mechanisms for the management of knowledge and improvement of the protocol, structure and teams, staff education and training, visual aids for the application of the protocol, activation of the protocol, communication with responsible public health institutions, hospitals or local authorities, attention to patients with suspected infection or who were already infected and measures for the prevention and control of infection. In total, 51 elements were considered (online suppl. appendix 1, see www.karger.com/doi/10.1159/000441793).

On the 28th October 2014, a preliminary audit was carried out (by J.J.M. and A.A.-A.) to appraise the tool's feasibility and adequacy as well as define the amount of time, the documents needed and the profile of the people involved in the audit. This pilot audit included the application of the checklist in a real context, and assessed the feasibility, comprehension, adequacy and reliability of 
Table 1. Results of the audit applying the EVD checklist for primary-care health-care centres

\begin{tabular}{|c|c|c|c|c|c|c|c|c|c|}
\hline Elements of the EVD protocol & \multicolumn{9}{|c|}{ Centres } \\
\hline $\begin{array}{l}2 \text { Mechanisms for managing knowledge and } \\
\text { improving the protocol }\end{array}$ & $0 / 2$ & $1 / 2$ & $1 / 2$ & $0 / 2$ & $1 / 2$ & $0 / 2$ & $1 / 2$ & $0 / 2$ & $1 / 2$ \\
\hline Structure and teams & $3 / 17$ & $0 / 17$ & $3 / 17$ & $1 / 17$ & $2 / 17$ & $3 / 17$ & $1 / 17$ & $2 / 17$ & $2 / 17$ \\
\hline Activation of the EVD protocol & $1 / 4$ & $1 / 4$ & $2 / 4$ & $1 / 4$ & $1 / 4$ & $2 / 4$ & $1 / 4$ & $1 / 4$ & $1 / 4$ \\
\hline $\begin{array}{l}7 \text { Communication with public health authorities } \\
\text { and other institutions }\end{array}$ & $0 / 2$ & $0 / 2$ & $0 / 2$ & $0 / 2$ & $1 / 2$ & $1 / 2$ & $1 / 2$ & $0 / 2$ & $1 / 2$ \\
\hline $\begin{array}{l}8 \text { Attention to patients with suspected infection or } \\
\text { who are already infected }\end{array}$ & $2 / 2$ & $2 / 2$ & $2 / 2$ & $1 / 2$ & $2 / 2$ & $2 / 2$ & $2 / 2$ & $2 / 2$ & $2 / 2$ \\
\hline Measures for the prevention and control of infection & $2 / 6$ & $2 / 6$ & $6 / 6$ & $3 / 6$ & $6 / 6$ & $5 / 6$ & $5 / 6$ & $4 / 6$ & $4 / 6$ \\
\hline
\end{tabular}

The figures express the number of areas of improvement/total number of checklist elements in centres 1-9.

the 51 elements. The meaning of the elements and the required response to satisfy each were clarified in the pilot study. This initial simulation of the audit suggested the need to modify the wording of 11 elements so as to improve clarity and avoid mistaken interpretations. In October 2014, the working group reviewed the proposal, and approved the checklist and audit procedure for the entire health district.

During November and December 2014, the first audits were carried out (by A.A.-A.) at the health centres. All elements were checked, verifying whether the procedures, equipment, training and visual aids had been tested and the health-care professionals were able to apply the EVD protocol. A member of the staff participated in the audit. The aim of the audit was explained and the checklist for applying the EVD protocol was presented in an appropriate way for patients and for health professionals. A member of staff of each centre was trained to use the checklist by themselves. The average duration of the audit was $3 \mathrm{~h} 40 \mathrm{~min}$. The outcomes analysis of the second audit was finished on the 3rd February 2015. The staff themselves carried out this second audit under supervision (by A.A.-A.). The procedure followed was identical in all cases.

\section{Results}

Audits were carried out at 9 health centres. Of the 51 checklist elements, 7-26 (14-51\%) required direct intervention in order to rectify deficits at the health centres (table 1).

In the course of the audits, a list of 'gaps' in correct application of the EVD protocol at each centre was elaborated. The worst gaps were related to: availability of materials ( $10 \%$ of the expected compliance), staff informa- tion and training (17\% success), communication with responsible agents and other institutions (17\% success) and the accessibility of the EVD protocol (19\% success). Examples of repeated failures were: no instruction sheet on the EVD protocol in accessible places ready for the initial contact with EVD patients, barriers to easy identification of changes in protocol, a lack of sets of working clothes being stored together with the personal protection equipment, no register of those professionals who had not yet received training, an absence of equipment for individual training and no up-to-date list of contact phone numbers for emergency transport or the local police. The audits also yielded 3 priority areas for improvement: (1) the incorporation of memory aids and instruction sheets for the correct application of protocol recommendations (e.g. on cleaning procedures for contaminated areas, the setting up and removal of personal protection equipment, critical questions that doctors should ask patients or the updating of risk zones identified in Africa), (2) the information that must be provided to patients and (where applicable) anyone who accompanied them and (3) help with how to proceed with people who might have had contact with the patient's family, e.g. those accompanying them to hospital, taxi and ambulance drivers, other patients in waiting rooms and health professionals. After the analysis of these deficits by the health centres, solutions were proposed and measures were implemented accordingly, as observed in the second audit. 


\section{Discussion}

In this study, we described the experience of the action we took in our health district to ensure proper implementation of the recommendations of the WHO and Spanish guidelines for Ebola. Anticipating potential failures was a necessary condition to ensure the adequate translation into actual practice of these guidelines. The audit and checklist were helpful in identifying gaps in preparedness for potential outbreaks, in reducing fears and in contributing to the proper training of health-care professionals in order to avoid potential mistakes.

The CDC in the USA disseminates a detailed hospital checklist for Ebola preparedness. This checklist provides practical suggestions to ensure that a hospital is able to detect possible Ebola cases, protect its employees and respond appropriately. The focus is to review and recognise what measures to take. There is no published checklist example for primary care. This is an experience in specific centres, so the focus is to review, step-by-step whether resources, equipment and professionals can cope with an Ebola suspect patient. There are 3 aims: the assurance of the primary care response capacity, protection of health professionals and support for them to be able to cope with the unprecedented threat.

The Ebola crisis generated confusion and worry among professionals with regard to the precautions to be taken as well as the safety and security of teams and procedures $[9,11]$. This situation may be similar to in other countries $[6,7]$. The checklist was designed to verify the applicability of the Ebola guidelines in actual practice, taking resources and procedures into consideration. Such checklists are especially useful because they help to eliminate the most problematic situations and they promote the use of memory aids and quality controls that help to reduce the incidence of errors [9]. The advantages of this approach lie in the greater specificity of the instructions and indications, which results in better implementation of the recommendations for action [17].

The majority of errors in the application of a protocol are related to the human factor [18]. These errors are involuntary and inadvertent, but they can have severe consequences. The mistake-proving approach (also termed Fail-Safe or Avoiding Inadvertent Errors) was used in this study. This checklist pursued design features that would prevent involuntary errors. Our approach contributed to a safer context by identifying the pressure involved in procedures that are unusual in primary care, the fear of contagion and the installations and organizations that are not designed for managing such cases, thereby making it more difficult to offer an appropriate response. Protocols do not 'implement themselves' [19], and it is necessary to foster their adaptation. Ebola is no exception.

\section{Conclusion}

The tool achieved the successful application of EVD protocol, assuring that primary care centres were able to handle an Ebola suspect patient. This tool contributes to generating a safer context.

\section{Acknowledgement}

The authors would like to thank the health centre coordinators and all the primary care and preventive medicine professionals, who, through their excellent work, contributed to making the health-care conditions safe for staff screening for viral haemorrhagic diseases such as Ebola.

\section{References}

1 Gerardo $\mathrm{CH}$, Nishiura H: Transmission dynamics and control of Ebola virus disease (EVD): a review. BMC Med 2014;12:196.

2 Bausch DG, Schwarz L: Outbreak of Ebola virus disease in Guinea: where ecology meets economy. PLoS Negl Trop Dis 2014; 8:e3056.

3 Parra JM, Salmerón OJ, Velasco M: The first case of Ebola virus disease acquired outside Africa. N Engl J Med 2014;371:2439-2440.

-4 Rodríguez-Caravaca G, Timermans R, ParraRamírez JM, et al: Ebola virus management committee. Health-care management of an unexpected case of Ebola virus disease at the
Alcorcón Foundation University Teaching Hospital. Enferm Infecc Microbiol Clin 2015; 33:228-232.

5 Lopaz MA, Amela C, Ordobas M, et al: First secondary case of Ebola outside Africa: epidemiological characteristics and contact monitoring, Spain, September to November 2014. Euro Surveill 2015;20:21003.

-6 SteelFisher GK, Blendon RJ, Lasala-Blanco N: Ebola in the United States - public reactions and implications. N Engl J Med 2015;373: 789-791.

7 Stephens DS, Ribner BS, Gartland BD, et al: Ebola virus disease: experience and decision making for the first patients outside of Africa. PLoS Med 2015;12:e1001857.

8 Klompas M, Diekema DJ, Fishman NO, et al: Ebola fever: reconciling Ebola planning with Ebola risk in U.S. hospitals. Ann Intern Med 2014;161:751-752.

-9 Rodríguez-Vega B, Amador B, Ortiz-Villalobos A, et al: The psychosocial response to the Ebola health emergency: experience in Madrid, Spain. Clin Infect Dis 2015;60:1866-1867.

10 Mosquera M, Melendez V, Latasa P: Handling Europe's first Ebola case: internal hospital communication experience. Am J Infect Control 2015;43:368-369. 
11 Mira JJ, Lorenzo S, Gea T, et al: Ebola's media outbreak: lessons for the future. Eur J Public Health 2015;25:188-189.

12 Simon N, Stockley SN, Rafi I: GPs and the Ebola patient: working safely in primary care. Br J Gen Pract 2014;64:636.

13 WHO: Global alert and response. Ebola virus 2014. http://www.who.int/csr/don/archive/ disease/ebola/en/ (accessed 29-10-2014).

14 Ministerio de Sanidad, Servicios Sociales e Igualdad, Dirección General de Salud Pública: Resumen del procedimiento de actuación frente a casos sospechosos de enfermedad por virus de Ebola (EVE) en relación con el brote de Guinea Conakry 26/08/2014. https://www. msssi.gob.es/profesionales/saludPublica/ ccayes/alertas Actual/ebola/docs/Procedimiento_Ebola_Profesionales_Agosto2014. pdf (accessed 29-10-2014).

15 US Department of Health and Human Services (DHHS): Centers for Disease Control and Prevention (CDC), Office of the Assistant Secretary for Preparedness and Response (ASPR): Hospital checklist for Ebola preparedness. http://www.floridahealth.gov/diseases-and-conditions/ebola/_documents/ hospital-checklist-ebola-preparedness.pdf (accessed 29-10-2014).
16 Carayon P, Wood KE: Patient safety: the role of human factors and systems engineering. Stud Health Technol Inform 2010;153:2346.

17 Michie S, Johnston M: Changing clinical behaviour by making guidelines specific. BMJ 2004;328:343-345.

18 Russ AL, Fairbanks RJ, Karsh B, et al: The science of human factors: separating fact from fiction. BMJ Qual Saf 2013;22:802-808.

19 Grimshaw JM, Thomas RE, MacLennan G, et al: Effectiveness and efficiency of guideline dissemination and implementation strategies. Health Technol Assess 2004;8:1-72. 